\title{
Correction to: Digital Health Solutions and Wearable Devices
}

Jennifer M. Joe, Jaydeo Kinikar, Monique Smith, Michael J. Carr, Ethan Bechtel, Stephen Randall, and Leah Ammerman

Corrections to: Chapter 2 in A. B. Bhatt (ed.), Healthcare Information Technology for Cardiovascular Medicine, Health Informatics, https://doi.org/10.1007/978-3-030-81030-6_2

This book was inadvertently published with a spelling error in the author's name of chapter 2. The correct name is Jaydeo Kinikar, this has been updated with this correction. 